\title{
COMMON FUZZY HYBRID FIXED POINT THEOREMS FOR A SEQUENCE OF FUZZY MAPPINGS
}

\author{
SHI CHUAN \\ Department of Applied Mathemaucs \\ Nanjing University of Science \& Technology \\ Nanjing, 210014, PEOPLE'S REPUBLIC OF CHINA
}

(Received September 28, 1995 and in revised form May 20, 1996)

\begin{abstract}
In this paper, we discuss the concepts of fuzzy hybrid fixed points, of $g-\Phi$-contractive type fuzzy mappings and common fuzzy hybrid fixed point theorems of a sequence of fuzzy mappings. Our theorems improve and generalize the corresponding recent important results.
\end{abstract}

KEY WORDS AND PHRASES: Fuzzy hybrid fixed point, common fuzzy hybrid fixed point, $g-\Phi$ contractive type fuzzy mapping, $g$-contractive type fuzzy mapping, sequence of fuzzy mappings. 1991 AMS SUBJECT CLASSIFICATION CODES: 54H25, 47H10.

\section{INTRODUCTION}

Heilpern [1] first introduced the concept of fuzzy mappings and proved fixed point theorems for contraction fuzzy mappings. Chang [2] introduced the concept of $\Phi$-contraction type fuzzy mappings, and proved a fixed point theorem, which is an extension of the result of Heilpern. Also, he obtained common fixed point theorems for a sequence of fuzzy mappings. Lee, et al [3-4] introduced the concept of $g$-contractive type fuzzy mappings, and proved a common fixed point theorem for sequence of fuzzy mappings on a complete metric linear space.

In this paper, we introduced $g-\Phi$-contractive type fuzzy mappings and defined the concept of the fuzz hybrid fixed point for fuzzy mappings, proved common fuzzy hybrid fixed point theorems for a sequence of fuzzy mappings on a complete metric space. Our theorems improve and generalize the recent important results of [1-4].

\section{PRELIMINARIES}

Throughout this paper let $(E, d)$ be a complete metric space, $C B(E)$ be a collection of all nonempty bounded closed subsets of $E$ and $C(E)$ be a collection of all non-empty compact subsets of $E$ Let $Z^{+}$be the set of all positive integers. A mapping $B: B \rightarrow[0,1]$ is called a fuzzy subset over $E$ We denote by $W(E)$ the family of all fuzzy subsets over $E$. Let $A \in W(E), \forall \alpha \in[0,1]$. Set $(A)_{\alpha}=\{x \in E: A(x) \geq \alpha\}$ is called the $\alpha$-cut set of $A$. A mapping $T: E \rightarrow W(E)$ is called fuzzy mapping over $E$.

DEFINITION 2.1. Let the function $\Phi:[0,+\infty)^{5} \rightarrow[0,+\infty)$. We say $\Phi$ satisfies the condition $\left(\Phi_{1}\right),\left(\Phi_{2}\right)$ or $\left(\Phi_{3}\right)$, if $\left(\Phi_{1}\right) \Phi$ is upper semi-continuous and non-decreasing for each variable $\left(\Phi_{2}\right) \Phi(t, t, t, a t, b t) \leq Q(t), \forall t \geq 0, a, b=0,1,2$, and $a+b=2$, where $Q(t):[0,+\infty) \rightarrow[0,+\infty)$, $Q(0)=0, Q(t)<t, \forall t>0 .\left(\Phi_{3}\right) \Phi(t, t, t, a t, b t) \leq r t$, where $r \in(0,1)$ is a constant, $a, b=0,1,2$ and $a+b=2$. 
DEFINITION 2.2. Let $T: E \rightarrow W(E)$. We say that $T: E \rightarrow W(E)$ satisfies the condition $A_{1}$ $\left(A_{2}\right)$. If there exists $\alpha(x): E \rightarrow(0,1]$ such that $\forall x \in E,(T x)_{\alpha(x)} \in C B(E)(C(E))$.

Let $T_{\imath}: E \rightarrow W(E)(i=1,2, \ldots)$. We say $T_{\imath}: E \rightarrow W(E)(i=1,2, \ldots)$ satisfies the condition $A_{1}$ $\left(A_{2}\right)$. If there exists a sequence of functions $a_{\imath}(x): E \rightarrow(0,1](i=1,2, \ldots)$ such that $\forall x \in E$, $(T i x)_{\alpha_{1}(x)} \in C B(E)($ or $C(E)$ ).

Let $T: E \rightarrow W(E)$ satisfies the condition $A_{1}$ (or $A_{2}$ ), $\forall x \in E, \tilde{T} x=(T x)_{\alpha(x)} \in C B(E)$. $\tilde{T}: E \rightarrow C B(E)$ is called the set-valued mapping induced by $T$.

DEFINITION 2.3. Let $g: E \rightarrow E$ be a single-valued mapping, $F: E \rightarrow W(E)$ and $G: E \rightarrow W(E)$ be two fuzzy mappings satisfying condition $A_{1}$. If, $\forall x, y \in E, u_{x} \in \tilde{F} x(\tilde{G} x)$ there exists $y, \in \tilde{G} y$ $(\tilde{F} y)$ such that

$$
\begin{gathered}
d\left(u_{x}, v_{y}\right) \leq \Phi\left(d(g(x), g(y)), d\left(g(x), g\left(u_{x}\right)\right), d\left(g(y), g\left(v_{y}\right)\right)\right. \\
\left.d\left(g(x), g\left(v_{y}\right)\right), d\left(g(y), g\left(u_{x}\right)\right)\right) .
\end{gathered}
$$

Then, we say that $F$ and $G$ satisfy the condition $B$.

DEFINITION 2.4. Let $F: E \rightarrow W(E), G: E \rightarrow W(E)$ be two fuzzy mappings satisfying the condition $A_{1}$. If for any $x, y \in E, u_{x} \in \tilde{F} x(\tilde{G} x)$ there exists $v_{y} \in \tilde{G} y(\tilde{F} y)$ such that

$$
d\left(u_{x}, v_{y}\right) \leq \Phi\left(d(x, y), d\left(x, u_{x}\right), d\left(y, v_{y}\right), d\left(x, v_{y}\right), d\left(y, u_{x}\right)\right) .
$$

Then, we say that $F, G$ satisfy the condition $C$.

DEFINITION 2.5. Let $F: E \rightarrow W(E)$ and $G: E \rightarrow W(E)$ be two fuzzy mappings satisfying the condition $A_{1}$. If for any $x, y \in E, u_{x} \in \tilde{F} x(\tilde{G} x)$ there exists $v_{y} \in \tilde{G} y(\tilde{F} y)$ such that

$$
H\left(\tilde{F}_{x}, \tilde{G}_{y}\right) \leq \Phi\left(d(x, y), d\left(x, \tilde{F}_{x}\right), d(y, \tilde{G} y), d(x, \tilde{G} y), d(y, \tilde{F} x)\right),
$$

where $d(x, \tilde{F} x)=\min _{p \in F_{x}} d(x, p)$ and $H$ is the Hausdorff metric induced by $d$, then, we say that $F$ and $G$ satisfy the condition $D$.

DEFINITION 2.6. Let $g: E \rightarrow E$ be a single-valued mapping, $F_{\imath}: E \rightarrow W(E)(i=1,2, \ldots)$ be a sequence of fuzzy mappings, if for any $i, j \in Z^{+}, F_{2}$ and $F_{j}$ satisfy conditions $A_{1}$ and $B$. Moreover, $\Phi$ in condition $B$ satisfies condition $\left(\Phi_{1}\right)$ and $\left(\Phi_{2}\right)$. Then we say $F_{2}: E \rightarrow W(E)(i=1,2, \ldots)$ be a $g-\Phi-$ contractive type sequence of fuzzy mappings. In particular, when $F_{2}=F_{\jmath}=F\left(\forall i, j \in Z^{+}\right)$we say $F: E \rightarrow W(E)$ be a $g-\Phi$-contractive type fuzzy mapping.

DEFINITION 2.7. Let $F: E \rightarrow W(E)$. If $P \in E$ such that $F p(p)=\max _{x \in E} F p(u)$, then $P$ is called a fixed point of $F$. Let $F_{2}: E \rightarrow W(E) \quad(i=1,2, \ldots)$. If $P \in E$ such that $\left(\bigcap_{i=1}^{+\infty} F i p\right)(p)=\max _{x \in E}\left(\bigcap_{i=1}^{+\infty} F i p\right)(u)$ then $P$ is called a common fixed point of $\left\{F_{2}\right\}$.

DEFINITION 2.8. Let $T: E \rightarrow E$ be a single-valued mapping and $F: E \rightarrow W(E)$ be a fuzzy mapping. If $P \in E$ such that $P=T p$ and $F p(p)=\max _{x \in E} F p(u)$, then $P$ is called a fuzzy hybrid fixed point of $T$ and $F$.

Let $T: E \rightarrow E$ be a single-valued mapping and $F_{\imath}: E \rightarrow W(E)(i=1,2, \ldots)$ be a sequence of fuzzy mappings. If $p \in E$ such that $p=T_{p}$ and $\left(\bigcap_{\imath=1}^{+\infty} F i p\right)(p)=\max _{x \in E}\left(\bigcap_{\imath=1}^{+\infty} F i p\right)(u)$, then $p$ is called a common fuzzy hybrid fixed point of $T$ and $\left\{F_{2}\right\}$.

\section{MAIN RESULTS}

THEOREM 3.1. Let $(E, d)$ be a complete metric space. Let:

(1) $T: E \rightarrow E$ be a single-valued continuous mapping such that $\forall x, y \in E$

$$
d(T x, T y) \leq d(x, T y)
$$


(2) $F_{\imath}: E \rightarrow W(E)(i=1,2, \ldots)$ be a $g$ - $\Phi$-contractive type sequence of fuzzy mappings, where $g: E \rightarrow E$ is a non-expansive mapping, $\alpha_{\imath}(x): E \rightarrow(0,1](i=1,2, \ldots)$ such that $\forall x \in E, T(F i x)_{\alpha_{1}(x)}$ $=(F i T x)_{\alpha_{1}(T x)}(i=1,2, \ldots)$.

(3) Let $\delta>1, x_{0} \in T(E), x_{1} \in\left(F_{1} x_{0}\right)_{\alpha_{1}\left(x_{0}\right)},\left\{t_{x}\right\}_{x=0}^{+\infty}$ be a sequence of nonnegative real numbers which is defined as follows

$$
\text { to }=0, t_{1}>d\left(x_{0}, x_{1}\right), t_{k+1}=t_{k}+Q\left(\delta\left(t_{k}-t_{k-1}\right)\right), k=1,2, \ldots
$$

If $\lim _{K \rightarrow \infty} t_{k}=t_{*}<+\infty$, then there exists $P \in E$ such that $P=T p$ and $\left(\bigcap_{\imath=1}^{+\infty} F i p\right)(p) \geq \min _{\imath \geq 1}\left\{\alpha_{\imath}(P)\right\}$, when $\alpha_{\imath}(x)=\max _{x \in E} F i x(u)(i=1,2, \ldots)$ be a sequence of functions satisfying the condition (2). Then there exists $P \in E$ such that $P=T p$ and $\left(\bigcap_{\imath=1}^{+\infty} F i p\right)(p)=\max _{x \in E}\left(\bigcap_{\imath=1}^{+\infty} F i p\right)(u)$, i.e $P$ be a common fuzzy hybrid fixed point of $T$ and $\left\{F_{2}\right\}$.

PROOF. Let $T(E)=\{x \mid x=T u, u \in E\}, F(E)=\{x \mid x=T x, x \in E\}$. It is obvious that $F(E) \subseteq T(E)$. Next we prove that $T(E) \subseteq F(E), \forall Z_{1} \in T(E), \exists u_{1} \in E$ with $Z_{1}=T u_{1}$, by (3.1), $0 \leq d\left(T z_{1}, T u_{1}\right) \leq d\left(z_{1}, T u_{1}\right)=d\left(z_{1}, z_{1}\right)=0, \therefore T z_{1}=T u_{1}=z_{1}, z_{1} \in F(E)$. Thus $T(E) \subseteq F(E)$, $T(E)=F(E)$.

We prove that $\forall x \in T(E), \tilde{F} i x \subseteq T(E)(i=1,2, \ldots)$. In fact, for $x \in T(E)=F(E)$ by $x=T x$, $T(F i x)_{\alpha_{i}(x)}=(F i T x)_{\alpha_{1}(T x)}(i=1,2, \ldots)$, we have $\tilde{F} i x=\tilde{F} i T x=(F i T x)_{\alpha_{1}(T x)}=T(F i x)_{\alpha_{1}(x)}$

$=T \tilde{F} i x \subseteq T(E)(i=1,2, \ldots)$ take $x_{0} \in T(E), x_{1} \in \tilde{F}_{1} x_{0} \subseteq T(E)$, by the condition $B$ and $g: E \rightarrow E$ be a non-expansive mapping, $\exists x_{2} \in \tilde{F}_{2} x_{1}$ such that

$$
\begin{aligned}
d\left(x_{1}, x_{2}\right) \leq & \Phi\left(d\left(g\left(x_{0}\right), g\left(x_{1}\right)\right), d\left(g\left(x_{0}\right), g\left(x_{1}\right)\right), d\left(g\left(x_{1}\right), g\left(x_{2}\right)\right)\right. \\
& \left.d\left(g\left(x_{0}\right), g\left(x_{2}\right)\right), \alpha\left(g\left(x_{1}\right), g\left(x_{1}\right)\right)\right) \\
\leq & \Phi\left(d\left(x_{0}, x_{1}\right), d\left(x_{0}, x_{1}\right), d\left(x_{1}, x_{2}\right), d\left(x_{0}, x_{2}\right), d\left(x_{1}, x_{1}\right)\right)
\end{aligned}
$$

for $x_{2} \in \tilde{F}_{2} x_{1}, \exists x_{3} \in \tilde{F}_{3} x_{2}$ such that

$$
d\left(x_{2}, x_{3}\right) \leq \Phi\left(d\left(x_{1}, x_{2}\right), d\left(x_{1}, x_{2}\right), d\left(x_{2}, x_{3}\right), d\left(x_{1}, x_{3}\right) d\left(x_{2}, x_{2}\right)\right) .
$$

Taking this procedure repeatedly, we can define a sequence $\left\{x_{s}\right\}$ in $T(E)$, satisfying $x_{s} \in \tilde{F}_{s x_{s-1}} \subseteq T(E), x_{s+1} \in \tilde{F}_{s+1} x_{s} \subseteq T(E)$, and

$$
d\left(x_{s}, x_{s+1}\right) \leq \Phi\left(d\left(x_{s-1}, x_{s}\right), d\left(x_{s-1}, x_{s}\right), d\left(x_{s}, x_{s+1}\right), d\left(x_{s-1}, x_{s+1}\right), d\left(x_{s}, x_{s}\right)\right) .
$$

We prove that $\left\{x_{s}\right\}_{s=0}^{+\infty}$ be convergent. First we prove the following inequality

$$
d\left(x_{n}, x_{n-1}\right) \leq \delta\left(t_{n}-t_{n-1}\right)(n=1,2, \ldots)
$$

for $n=1, d\left(x_{1}, x_{0}\right)<t_{1}=t_{1}-t_{0}<\delta\left(t_{1}-t_{0}\right),(3.4)$ is true. Suppose that $n=k$. (3.4) is true, i.e. $d\left(x_{k}, x_{k-1}\right) \leq \delta\left(t_{k}-t_{k-1}\right) \quad$ We prove that it remains true for $n=k+1$, when $n=k+1$, by $\left(\Phi_{1}\right),\left(\Phi_{2}\right),(3.2),(3.3), d\left(x_{k-1}, x_{k+1}\right) \leq d\left(x_{k-1}, x_{k}\right)+d\left(x_{k}, x_{k+1}\right)$, and it is easy to prove that $d\left(x_{k+1}, x_{k}\right) \leq d\left(x_{k-1}, x_{k}\right)$, we have

$$
\begin{aligned}
d\left(x_{k+1}, x_{k}\right) & \leq \Phi\left(d\left(x_{k}, x_{k-1}\right), d\left(x_{k}, x_{k-1}\right), d\left(x_{k}, x_{k+1}\right), d\left(x_{k-1}, x_{k+1}\right), d\left(x_{k}, x_{k}\right)\right) \\
& \leq \Phi\left(d\left(x_{k}, x_{k-1}\right), d\left(x_{k}, x_{k-1}\right), d\left(x_{k-1}, x_{k}\right), 2 d\left(x_{k-1}, x_{k}\right), 0\right) \\
& \leq \Phi\left(\delta\left(t_{k}-t_{k-1}\right), \delta\left(t_{k}-t_{k-1}\right), \delta\left(t_{k}-t_{k-1}\right), 2 \delta\left(t_{k}-t_{k-1}\right), 0\right) \\
& \leq Q\left(\delta\left(t_{k}-t_{k-1}\right)\right)=t_{k+1}-t_{k}<\delta\left(t_{k+1}-t_{k}\right) .
\end{aligned}
$$

Thus (3.4) remains true for $n=k+1$. This completes the proof of (3.4).

By $\lim _{k \rightarrow \infty}=t_{*}<+\infty$ and (3.4) $d\left(x_{k+m}, x_{k}\right) \leq \sum_{\jmath=k}^{k+m-1} d\left(x_{\jmath+1}, x_{\jmath}\right) \leq \delta \sum_{\jmath=k}^{k+m-1}\left(t_{\jmath+1}-t_{\jmath}\right)=\delta\left(t_{k+m}-t_{k}\right)$. Thus $\{x s\}_{s=0}^{+\infty}$ be a Cauchy sequence in $T(E)$. Since $(T(E), d)$ is a complete metric space, therefore $\exists$ $P \in E$ such that $\lim _{s \rightarrow \infty} x_{s}=P, \because P \in T(E)=F(E), \therefore P=T p$. Next, we prove that $P \in \bigcap_{i=1}^{+\infty} \tilde{F} i p, \forall$ 
$m \in Z^{+}, \because x_{s} \in \tilde{F} s x_{s-1}(n=1,2, \ldots)$ by the condition $B$ and $g: E \rightarrow E$ be a nonexpansive mapping, $\exists v, \in \tilde{F} m p$ such that

$$
d\left(x_{s}, v_{s}\right) \leq \phi\left(d\left(x_{s-1}, p\right), d\left(x_{s-1}, x_{s}\right), d\left(p, v_{s}\right), d\left(x_{s-1}, v_{s}\right), d\left(p, x_{s}\right)\right)
$$

by the condition $A_{1}, \tilde{F} m p=(F m p)_{\alpha_{m}(p)} \in C B(E), \tilde{F} m p$ be a non-empty bounded closet set of $E, v_{s} \in \tilde{F} m p$. Thus $\left\{d\left(v_{s}, p\right)\right\}$ be a bounded sequence of real numbers. Therefore, there exists $\left\{d\left(v_{s,}, p\right)\right\} \subseteq\left\{d\left(v_{s}, p\right)\right\}$ satisfies $\lim _{j \rightarrow \infty} d\left(v_{s}, p\right)=d$, by (3.5) and $d\left(v_{s,}, x_{s_{s}-1}\right) \leq d\left(v_{s_{s}}, p\right)+d\left(x_{s_{s}-1}, p\right)$, we have

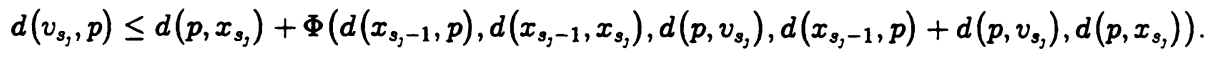

Let $j \rightarrow+\infty$, by $d\left(v_{s}, p\right) \rightarrow d, x_{s} \rightarrow p,\left(\Phi_{1}\right),\left(\Phi_{2}\right)$, we have, when $d \neq 0$

$$
d \leq+\Phi(0,0, d, 0+d, 0) \leq Q(d)<d .
$$

This is a contradiction, therefore, $d=0$, i.e. $\lim _{g \rightarrow \infty} v_{s_{s}}=p$, by $v_{s,} \in \tilde{F} m p$ and $v_{s_{s}} \rightarrow p, \therefore p \in \tilde{F} m p=$ $(F m p)_{\alpha_{m}(p)}\left(\forall m \in z^{+}\right)$i.e. $F m p(p) \geq \alpha_{m}(p)(m=1,2, \ldots)$. Thus $F m p(p) \geq \min _{i \geq 1}\left\{\alpha_{i}(p)\right\}(m=1,2, \ldots)$, $\left(\bigcap_{m=1}^{+\infty} F m p\right)(p)=\min _{i \geq 1}\left\{\alpha_{i}(p)\right\}$.

When $\alpha_{\imath}(x)=\max _{x \in E} F i x(u)(i=1,2, \ldots)$. Then $\left(\bigcap_{i=1}^{+\infty} F i p\right)(p) \geq \min _{i \geq 1}\left\{\alpha_{\imath}(p)\right\} \geq \min _{i \geq 1} \max _{\mu \in E} F i p(u) \geq$ $\min _{i \geq 1} F i p(u)=\left(\bigcap_{i=1}^{+\infty} F i p\right)(u), \forall u \in E$. Thus $\left(\bigcap_{i=1}^{+\infty} F i p\right)(p) \geq \max _{\mu \in E}\left(\bigcap_{i=1}^{+\infty} F i p\right)(u) \geq\left(\bigcap_{i=1}^{+\infty} F i p\right)(p)$. $\therefore\left(\bigcap_{\imath=1}^{+\infty} F i p\right)(p)=\max _{\mu \in E}\left(\bigcap_{\imath=1}^{+\infty} F i p\right)(u)$, i.e. $p$ be common fixed point of $\left\{F_{i}\right\}$, by $p \in T(E)=F(E)$, $p=T p$. Thus $p$ be a common fuzzy hybrid fixed point of $T$ and $\left\{F_{2}\right\}$.

COROLLARY 3.1. Let $(E, d), T: E \rightarrow E$ and $F_{2}: E \rightarrow W(E)(i=1,2, \ldots)$ satisfy the conditions of Theorem 3.1. Moreover $\Phi$ satisfies the condition $\left(\Phi_{3}\right)$, then the conclusion of Theorem 3.1 remains true.

PROOF. Taking $t_{0}=0, x_{0} \in T(E), x_{1} \in \tilde{F}_{1} x_{0}, t_{1}>d\left(x_{0}, x_{1}\right)$. We define a sequence of nonnegative real numbers $\left\{t_{k}\right\}_{k=0}^{+\infty}$ as follows:

$$
t_{k+1}=t_{k}+r \delta\left(t_{k}-t_{k-1}\right), k=1,2, \ldots
$$

where $\delta>1$ and $\delta r<1, r$ be a constant in the condition $\left(\Phi_{3}\right)$. It follows from (3.6)

$$
t_{k+1}-t_{k}=r \delta\left(t_{k}-t_{k-1}\right)=\cdots=(r \delta)^{k} t_{1} .
$$

Therefore we have $\lim _{k \rightarrow \infty} t_{k}=\lim _{k \rightarrow \infty} \sum_{\imath=1}^{k}\left(t_{\imath}-t_{i-1}\right)=\frac{t_{1}}{1-r \delta}<+\infty$. The conclusion of Corollary 31 follows from Theorem 3.1 immediately.

COROLLARY 3.2. Let $(E, d), T: E \rightarrow E$ satisfies the condition of Theorem 3.1 Let $F_{2}: E \rightarrow W(E)(i=1,2, \ldots)$ for $\alpha_{i}(x): E \rightarrow(0,1](i=1,2, \ldots)$ satisfies the condition $A_{2}$ and $\forall$ $x \in E, T(F i x)_{\alpha_{2}(x)}=(F i T x)_{\alpha_{1}(T x)}(i=1,2, \ldots)$. Moreover, for any $i, j \in z^{+}, x, y, \in E, u_{x} \in \tilde{F} i x, \exists$ $v_{y} \in F j y$ such that

$$
\begin{aligned}
d\left(u_{x}, v_{y}\right) \leq & g \max \left\{d(g(x), g(y)), d\left(g(x), g\left(u_{x}\right)\right)\right. \\
& \left.d\left(g(y), g\left(v_{y}\right)\right), \frac{1}{2}\left[d\left(g(x), g\left(v_{y}\right)\right)+d\left(g(y), g\left(u_{x}\right)\right)\right]\right\}
\end{aligned}
$$

where $q \in(0,1)$ is a constant, $g: E \rightarrow E$ be a non-expansive mapping. Then the conclusion of Theorem 3.1 remains true. 
PROOF. Taking $\Phi\left(t_{1}, t_{2}, t_{3}, t_{4}, t_{5}\right)=q \max \left\{t_{1}, t_{2}, t_{3}, \frac{1}{2}\left(t_{4}+t_{5}\right)\right\}$, we have $\Phi(t, t, t, a t, b t)=q t$, where $a, b=0,1,2$ and $a+b=2$. It is easy to see that $\Phi$ satisfies the condition $\left(\Phi_{1}\right)$ and $\left(\Phi_{3}\right)$, therefore the conclusion follows from Corollary 3.1 directly.

THEOREM 3.2. Let $(E, d)$ and $T: E \rightarrow E$ satisfy the condition of Theorem 3.1. Let $F_{\imath}: E \rightarrow W(E)(i=1,2, \ldots)$ for $\alpha_{2}(x): E \rightarrow(0,1](i=1,2, \ldots)$ satisfy the condition $A_{1}$ and $\forall$ $x \in E, T(F i x)_{\alpha_{2}(x)}=(F i T x)_{\alpha_{1}(T x)}(i=1,2, \ldots)$. Moreover for any $i, j \in Z^{+}, x, y \in E, u_{x} \in \tilde{F}_{i x}, \exists$ $v_{y} \in \tilde{F} j y$ such that

$$
\begin{aligned}
d\left(u_{x}, v_{y}\right) \leq & \alpha_{1} d\left(g(x), g\left(u_{x}\right)\right)+\alpha_{2} d\left(g(y), g\left(v_{y}\right)\right)+\alpha_{3} d\left(g(y), g\left(u_{x}\right)\right) \\
& +\alpha_{r} d\left(g(x), g\left(v_{y}\right)\right)+\alpha_{5} d(g(x), g(y))
\end{aligned}
$$

where $g: E \rightarrow E$ be a non-expansive mapping, $\alpha_{\imath}>0(i=1,2, \ldots, 5), \alpha_{1}+\alpha_{2}+\ldots+\alpha_{5}<1$ and $\alpha_{3} \geq \alpha_{4}$. Then the conclusion of Theorem 3.1 remains true.

PROOF. By proof of Theorem 3.1, $T(E)=F(E)$, and $\forall x \in T(E), \tilde{F} i x \subseteq T(E)(i=1,2, \ldots)$, by (3.8) and $g: E \rightarrow E$ be a non-expansive mapping, the same as the proof of Theorem 3.1 We can define a sequence $\left\{x_{s}\right\} \subseteq T(E)$, such that $x_{s+1} \subseteq \tilde{F}_{s+1} x_{s} \subseteq T(E)$. Moreover

$$
\begin{aligned}
d\left(x_{s}, x_{s+1}\right) & \leq \alpha_{1} d\left(x_{s-1}, x_{s}\right)+\alpha_{2}\left(x_{s}, x_{s+1}\right)+\alpha_{3} d\left(x_{s}, x_{s}\right) \\
& +\alpha_{4} d\left(x_{s-1}, x_{s+1}\right)+\alpha_{5} d\left(x_{s-1}, x_{s}\right) \\
& \leq \alpha_{1} d\left(x_{s-1}, x_{s}\right)+\alpha_{2} d\left(x_{s}, x_{s+1}\right)+\alpha_{4} d\left(x_{s-1}, x_{s}\right) \\
& +\alpha_{4} d\left(x_{s}, x_{s+1}\right)+\alpha_{5} d\left(x_{s-1}, x_{s}\right) .
\end{aligned}
$$

Therefore

$$
\alpha\left(x_{s}, x_{s+1}\right) \leq \frac{\alpha_{1}+\alpha_{4}+\alpha_{5}}{1-\alpha_{2}-\alpha_{4}} d\left(x_{s-1}, x_{s}\right)
$$

$\because \alpha_{3} \geq \alpha_{4}>0, \alpha_{1}+\cdots+\alpha_{5}<1$. Thus $r=\frac{\alpha_{1}+\alpha_{4}+\alpha_{5}}{1-\alpha_{2}-\alpha_{4}}<1$, we have

$$
d\left(x_{s}, x_{s+1}\right) \leq r d\left(x_{s-1}, x_{s}\right) \leq r^{2} d\left(x_{s-2}, x_{s}\right) \leq \cdots \leq r^{s} d\left(x_{0}, x_{1}\right) .
$$

By (3.10), it is easy to see that $\left\{x_{s}\right\}_{s=1}^{+\infty}$ is a Cauchy sequence in $T(E)$. Thus $\exists p \in T(E)$, such that $\lim _{s \rightarrow \infty} x_{s}=p$. Next, we prove that $p \in \bigcap_{m=1}^{+\infty} \tilde{F}_{m p}, \forall m \in Z^{+}$, for $x_{s} \in \tilde{F}_{s} x_{s-1}(n=1,2, \ldots)$, by assumption, $\exists v_{s} \in \tilde{F} m p$ such that

$$
\begin{aligned}
d\left(x_{s}, v_{s}\right) \leq & \alpha_{1} d\left(x_{s-1}, x_{s}\right)+\alpha_{2} d\left(p, v_{s}\right)+\alpha_{3} d\left(p, x_{s}\right) \\
+ & \alpha_{4} d\left(x_{s-1}, v_{s}\right)+\alpha_{5} d\left(x_{s-1}, p\right) \\
\leq & \alpha_{1} d\left(x_{s-1}, x_{s}\right)+\alpha_{2} d\left(p, x_{s}\right)+\alpha_{2} d\left(x_{s}, v_{s}\right) \\
& \alpha_{3} d\left(p, x_{s}\right)+\alpha_{4} d\left(x_{s-1}, x_{s}\right)+\alpha_{4} d\left(x_{s}, v_{s}\right)+\alpha_{5} d\left(x_{s-1}, p\right) .
\end{aligned}
$$

Thus we have

$$
\begin{aligned}
\left(1-\alpha_{2}-\alpha_{4}\right) d\left(x_{s}, v_{s}\right) \leq & \alpha_{1} d\left(x_{s-1}, x_{s}\right)+\alpha_{2} d\left(p, x_{s}\right) \\
& +\alpha_{3} d\left(p, x_{s}\right)+\alpha_{4} d\left(x_{s-1}, x_{s}\right)+\alpha_{5} d\left(x_{s-1}, p\right) .
\end{aligned}
$$

We have $d\left(x_{s}, v_{s}\right) \rightarrow 0(n \rightarrow+\infty)$. Thus $d\left(v_{s}, p\right) \leq d\left(v_{s}, x_{s}\right)+d\left(x_{s}, p\right) \rightarrow 0(n \rightarrow+\infty), \therefore \lim _{s \rightarrow \infty} v_{s}=p$, by $v_{s} \in \tilde{F} m p \in C B(E)$. Therefore $p \in \tilde{F} m p\left(\forall m \in z^{+}\right)$. By $p=T p$ and $p \in \bigcap_{m=1}^{+\infty} \tilde{F} m p$, the same as the proof of Theorem 3.1, we obtain the conclusion of Theorem 3.1.

When $T=I$ is the identity operator on $E$, we obtain the following result.

COROLLARY 3.3. Let $(E, d)$ and $F_{2}: E \rightarrow W(E)(i=1,2, \ldots)$ satisfy the conditions of Theorem 3.2. Then there exists $p \in E$ such that $\left(\bigcap_{\imath=1}^{+\infty} F i p\right)(p) \geq \min _{\imath \geq 1}\left\{\alpha_{\imath}(p)\right\}$, when $\alpha_{i}(x)=\max _{x \in E} F i x(u)$ $(i=1,2, \ldots)$ satisfies corresponding conditions, $p$ is a common fixed point of $\left\{F_{2}\right\}$. 
REMARK 3.1. When $\alpha_{3}=\alpha_{4}$ in the condition (3.8) of Theorem 3.2, Theorem 3.2 is a special case of Corollary 3.2. Corollary 3.3 is an improvement and generalized version of Theorem 3.1 of [4] and Theorem 3.10 of [3]. In Theorem 3 of [2], if $\left\{F_{i}\right\}$ for $\left\{\alpha_{i}(x)\right\}$ satisfy condition $A_{2}$, then Theorem 3 of [2] is a special case of Theorem 3.1 of this paper. In fact, when $T=I$ and $g=I$ are identity operators on $E$, by the theorem of Nadler [5], it is easy to see the condition $D$ implies the condition $C$.

\section{REFERENCES}

[1] HEILPERN, S., Fuzzy mappings and fixed point theorem, J. Math. Anal. Appl. 83 (1981), 566569 .

[2] SHIH-SEN, C., Fixed degree for fuzzy mappings and a generalization of Ky Fan's theorem, Fuzzy Sets and Systems, 24 (1984), 103-112.

[3] LEE, B.S. and CHO, S.J., Common fixed point theorems for sequences of fuzzy mappings, Internat. J. Math. \& Math. Sci., 17, 3 (1994), 423-428.

[4] LEE, B.S., LEE, G.M., CHO, S.J. and KIM, D.S., Generalized common fixed point theorems for a sequence of fuzzy mappings, Internat. J. Math. \& Math. Sci. 17, 3 (1994), 437-440.

[5] NADLER, S.B. Jr., Muli-valued contraction mappings, Pacific J. Math. 30 (1969), 475-488. 


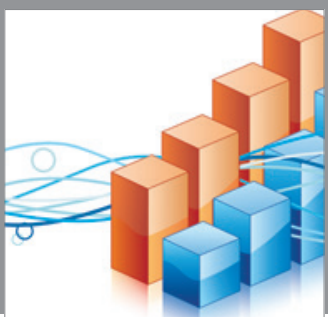

Advances in

Operations Research

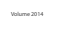

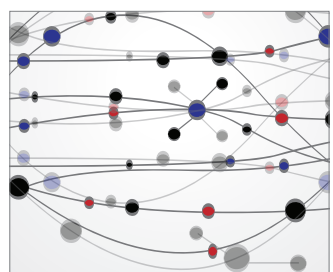

\section{The Scientific} World Journal
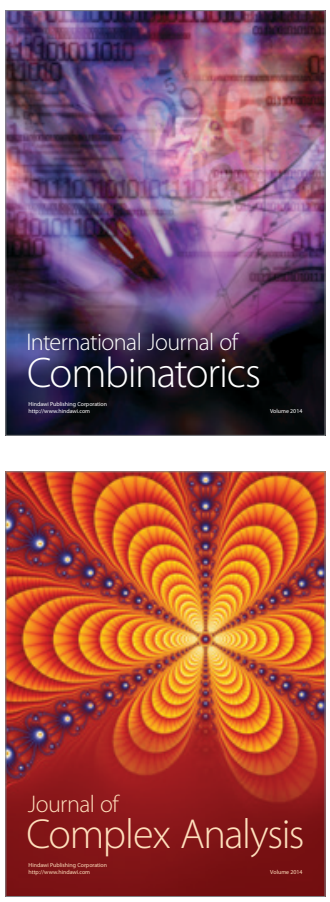

International Journal of

Mathematics and

Mathematical

Sciences
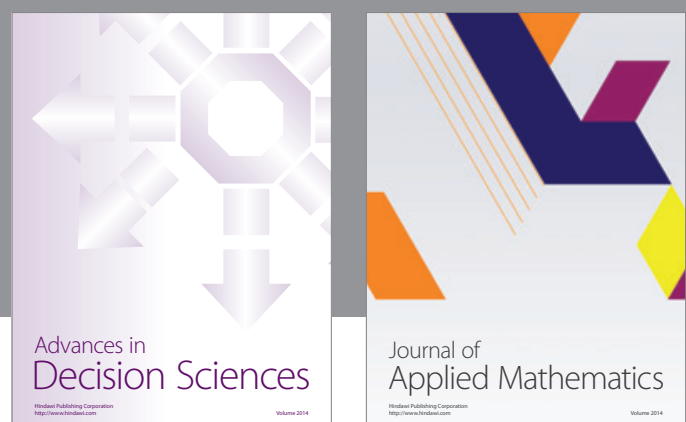

Journal of

Applied Mathematics
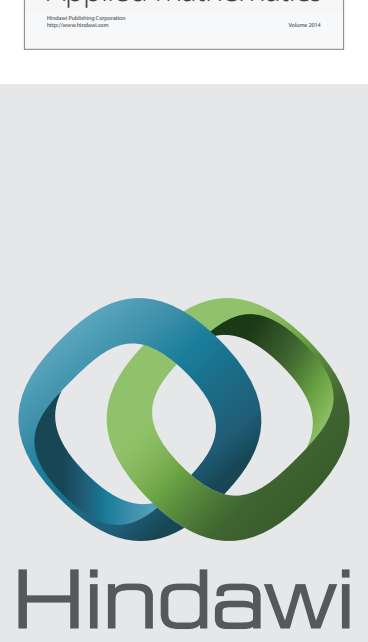

Submit your manuscripts at http://www.hindawi.com
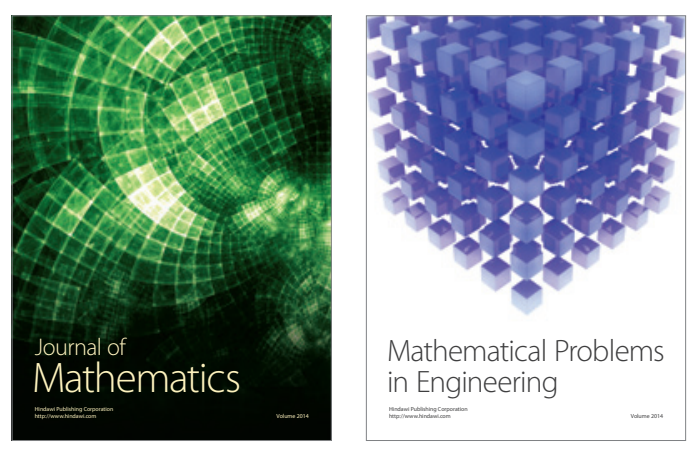

Mathematical Problems in Engineering
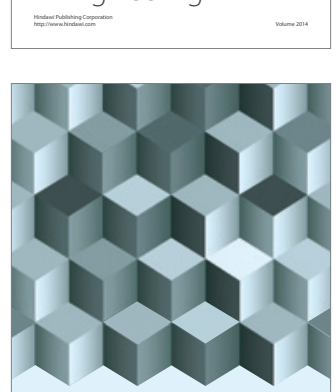

Journal of

Function Spaces
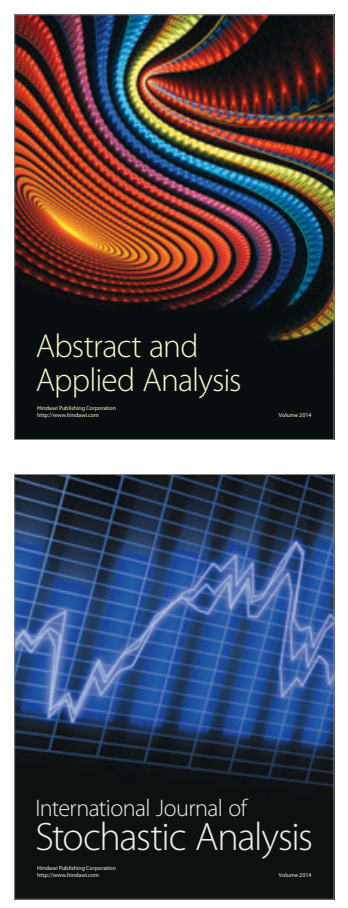

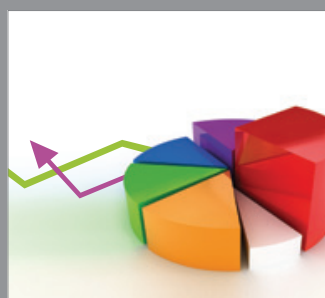

ournal of

Probability and Statistics

Promensencen
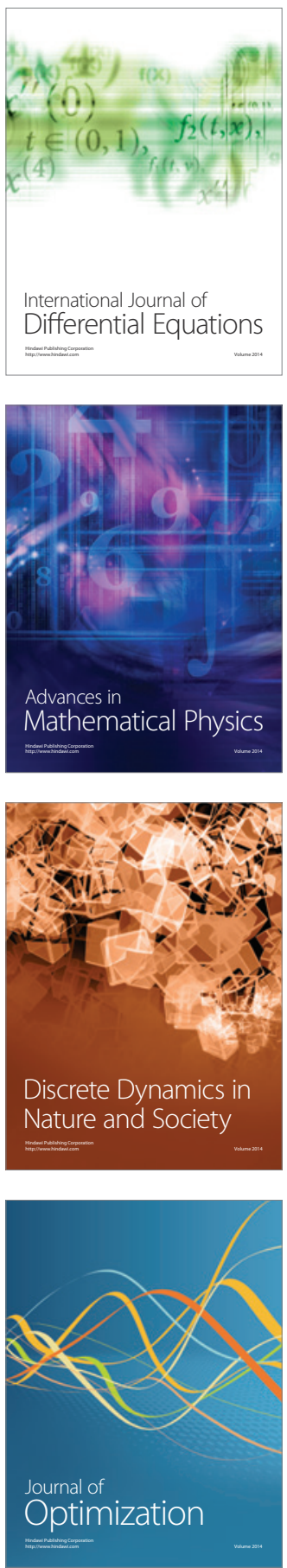